\title{
Calidad de servicio en hotel Ysapy de la ciudad de Pilar, desde la perspectiva del cliente, 2021
}

\author{
Rodrigo Daniel Amarilla Delvalle \\ rodrigoamarilla2017@gmail.com \\ Universidad Nacional de Pilar \\ Raúl Merlos Martínez \\ raulmerlos06@gmail.com \\ Universidad Nacional de Pilar
}

\section{RESUMEN}

El presente estudio sobre servicio hotelero, busca conocer calidad de servicio del Hotel Ysapy de la ciudad de Pilar, desde la perspectiva del usuario, mediante la aplicación de cuestionario estandarizado del modelo Servequal que abarca cinco dimensiones: fiabilidad, capacidad de repuestas, seguridad, empatía y elementos tangibles, metodológicamente adquiere un diseño cuantitativo de alcance descriptivo, temporalmente transversal, abarcó una muestra de 100 usuarios, los datos fueron recogidos entre los meses de marzo, abril y mayo del año 2021. El estudio identifica el perfil del usuario y en promedio general resultante en las cinco dimensiones evaluadas indica que la calidad del servicio oscila entre un nivel de bueno a excelente. Reporta aspectos puntuales de la gestión del servicio que deben sostenerse y otros a ser incorporados en planes de mejora continua como estrategia de gestión orientada a consolidar la calidad del servicio.

Palabras claves: calidad de servicio; modelo servqual; hotelería 


\title{
Quality of service at hotel Ysapy in the city of Pilar, from the customer's perspective, 2021
}

\begin{abstract}
This study on hotel service seeks to know the quality of service of the Ysapy Hotel in the city of Pilar, from the user's perspective, through the application of a standardized questionnaire of the Servequal model that covers five dimensions: reliability, response capacity, security, empathy and tangible elements, methodologically it acquires a quantitative design of descriptive scope, temporally transversal, covered a sample of 100 users, the data was collected between the months of March, April and May of the year 2021. The study identifies the user's profile and on average The overall result in the five dimensions evaluated indicates that the quality of the service ranges from a level of good to excellent. It reports specific aspects of service management that must be sustained and others to be incorporated into continuous improvement plans as a management strategy aimed at consolidating the quality of the service.
\end{abstract}

Keywords: quality of service; servqual model; hospitality

Artículo recibido: 02 Setiembre. 2021 Aceptado para publicación: 30 Setiembre. 2021 Correspondencia: rodrigoamarilla2017@gmail.com Conflictos de Interés: Ninguna que declarar 


\section{INTRODUCCIÓN}

La Ciudad de Pilar, capital de Departamento de Neembucú República del Paraguay, distante $358 \mathrm{~km}$ al sur de Asunción, con aproximadamente 32.810 habitantes, según proyecciones del (Instituto Nacional de Estadística, 2018), en la ciudad existen registrados y funcionados 11 establecimientos de servicios hoteleros, de los cuales 5 establecimientos están ubicados en la zona céntrica correspondiente al territorio de Barrio Obrero, y poseen características similares en cuando a la infraestructura física y servicio, de los cuales se seleccionó a un establecimiento como objeto de estudio que por consideraciones éticas del estudio se denomina hotel Ysapy.

El hotel Ysapy cuenta con 10 años de servicio en el mercado de hospitalidad, funciona en la estructura edilicia moderna diseñada para su funcionamiento para atención, cuenta en total con 18 habitaciones, con servicio de: el desayuno, TV cable, Wifi, ambiente climatizado y estacionamiento propio, atiende las 24 horas de día, con atención personalizada y virtual. La mayoría de los clientes del establecimiento son personas quienes vienen a la ciudad con fines comerciales o turísticas. Aproximadamente albergan por semana entre 40 a 50 clientes, en mayor número son jóvenes trabajadores como: proveedores de productos para la ciudad o el departamento, fiscalizadores de obras, vendedores y otros que poseen una buena base de estudios académicos y en gran parte ya son clientes del hotel, y ya tienen contacto frecuente con la empresa y en algunos casos ya son beneficiados con descuentos especiales (Según datos del propietario).

El departamento de Ñeembucú posee atractivos turísticos de naturaleza, cultural e histórico (zona de humedales con diversidad biológica, zonas históricas epicentro de la guerra de la triple alianza, fiestas emblemáticas, playas, torneos de pesca) que atraen a visitantes que usufructúan los servicios hoteleros (Riveros, et al., 2019, Senatur, 2020).

En el mercado de servicios de hospitalidad están relacionados la competencia y la mejora de la calidad de las empresas, el servicio de excelencia de los hoteles es esencial, contribuye de gran manera para el desarrollo sostenible de los destinos turísticos y posicionamiento de la ciudad (Mansalve Castro \& Hernández Rueda, 2015).

Las empresas hoteleras deben hacer el esfuerzo necesario y efectivo cómo implementar estrategias acordes a las necesidades y exigencias de usuarios para posicionarse como marca de calidad, mantener la competitividad y sostenerse en el tiempo (Vavra, 2000). Un factor clave para lograrlo es conocer el perfil, necesidad y expectativa de clientes, poner a 
disposición productos, servicios, modo de atención comunicación y mejoras continuas, lo redundara en la satisfacción y fidelización de usuarios, entre otros aspectos positivos (Thompson, 2005). No tomar en cuenta al usuario en contexto de competencia pone en riesgo el éxito económico y la sostenibilidad de la empresa (Arias Muñoz, 2019).

Evaluar la calidad de servicios brindados en hoteles desde la perspectiva del usuario es una herramienta que permite identificar brecha entre el servicio brindado y la expectativa del cliente y al mismo tiempo una estrategia para generar insumos orientada a la toma de decisiones oportunas a beneficio del negocio.

Este estudio se propone conocer y analizar la calidad de servicio del HotelYsapy de la ciudad de Pilar, en cinco componentes vinculados a recursos, gestión y atención en los aspectos: tangibles, fiabilidad, empatía, seguridad y capacidad de respuesta, para los fines de este estudio fueron modificados algunos indicadores considerando la característica del objeto de estudio en el contexto local utilizando el modelo Servqual.

La investigación adquiere relevancia considerando que Senatur Paraguay establece como indicadores para evaluación de la calidad de los servicios hoteleros los siguientes aspectos relacionados con este estudio: la gestión operativa, servicios e infraestructura. Partiendo de estos puntos importantes, pretendemos evaluar la calidad de servicio del Hotel Ysapy de la ciudad de Pilar, utilizando el cuestionario adecuado al Modelo Servequal. Dicho modelo nos permite analizar la percepción de los usuarios sobre los servicios que brinda actualmente el hotel, en relación a los criterios de: elementos tangibles, fiabilidad, capacidad de respuesta, seguridad y empatía (Girón, 2016).

En cuanto a lo económico y social, con el estudio permitirá conocer la calidad de servicio del Hotel Ysapy y la posibilidad para los encargados de corregir algunas fallas existentes para garantizar la satisfacción de los huéspedes. Ya que es un establecimiento que está instalado en el mercado y es importante la sostenibilidad del servicio. Además, el estudio puede ser replicado a otros servicios locales como instrumento de evaluación de la calidad. Así también, los resultados del estudio pueden ser utilizados como referencia para otros estudios, quienes desean profundizar más sobre el tema. 


\section{Calidad de servicio}

\section{Respecto a los creadores del instrumento.}

Valarie Zeithaml es una profesora e investigadora galardonada y una pionera del marketing de servicios reconocida internacionalmente, ha investigado las expectativas de los clientes en más de 50 industrias (UNC, 2021).

Por otro lado Parasuraman es considerado una de las figuras más influyentes en el campo del marketing de servicios y la calidad del servicio, y es ampliamente conocido por su trabajo en SERVQUAL, ES-QUAL y el Technology Readiness Index (TRI (ROCKBRIDGE, 2021). Por último, el Dr. Leonard L. Berry es un distinguido profesor de Marketing y ocupa un puesto en Ventas y Liderazgo en Marketing en la Escuela de Negocios de Texas, AM University (GROWTH INSTITUTE, 2019)

\section{Dimensiones de Servqual según Wigodski Sirebrenik( 2003)}

Percepción de las necesidades del cliente, el cliente tiene ciertas necesidades, que son percibidas por el sistema para posterior realización del servicio.

Expectativas del cliente, define lo que espera el cliente del servicio, estas expectativas son formadas por series de factores como: comunicación boca a boca, información externa, experiencia pasadas o por necesidades específicas. Desde este punto se puede apreciar ciertos necesidades o diferencias, cuando el cliente emite una percepción. Estas cinco

\section{dimensiones del modelo Servqual son:}

1) Fiabilidad o continuidad de la prestación del servicio prometido de modo cuidadoso y estable en el tiempo

2) Capacidad de respuesta: disposición del personal para prestar ayuda y servicio rápido a los usuarios;

3) Seguridad: atención y habilidades dispensadas por los empleados para inspirar credibilidad y confianza;

4) Empatía: capacidad para comprender la perspectiva del usuario;

5) Aspectos tangibles: características de las instalaciones físicas, equipos, personal y material de comunicación.

\section{ESTRATEGIAS METODOLÓGICAS}

El tipo de investigación es de carácter exploratorio y cuantitativo. La técnica de recolección de datos es mediante encuesta y el instrumento utilizado es cuestionario adecuado al Modelo Servqual, teniendo como muestra a 100 usuarios del hotel Ysapy. El tiempo de duración de 
la fase empírica o de recolección de datos fue de 3 meses (desde 01-03-2021 al 30-05-2021), por el tiempo requerido para completar la cantidad de respuestas de usuarios.

El proceso metodológico del estudio fue de la siguiente manera, en primer lugar, se revisó el cuestionario Servqual, se adecuo a la realidad. Quedo estructurado en total con 22 preguntas de las 5 dimensiones del modelo Servqual. Dimensión de aspecto tangible con 4 preguntas, fiabilidad 5 preguntas, capacidad de respuesta 4 preguntas, seguridad 4 preguntas y empatía con 5 preguntas.

El modelo Servqual evalúa la respuesta en forma cualitativa y cuantitativa, la valoración cuantitativa determina el juicio cualitativo de "deficiente", "aceptable", "regular", "bueno" y "excelente".

Deficiente: indica un nivel muy bajo de la calidad, que no satisface la necesidad de los usuarios y que requiere de mejoría inmediata.

Aceptable: indica un nivel bajo, requiere de mejoría inmediata, pero satisface en partes la necesidad de los clientes

Regular: indica un nivel medio de la calidad del servicio,

Bueno indica un nivel alto de la calidad del servicio, pero requiere de algunos ajustes para llegar a la excelencia; y la Excelencia que representa el nivel óptimo de la calidad del servicio.

En consideraciones éticas, se aplica el principio de confidencialidad resguardando la identidad del establecimiento estudiado, así como la identidad de los encuestados.

Luego se solicitó el permiso correspondiente para la realización del estudio mediante una nota dirigida al propietario del establecimiento hotelero, considerando la respuesta favorable a la petición, se realizó una entrevista al propietario, quien brindo algunos datos sobre el funcionamiento del hotel, la clientela y otros, también facilito la lista de registros de los clientes que usufructúan los servicios.

Posteriormente se procedió a la realización de la prueba piloto del cuestionario, en especial de Google Drive. Luego se procedió a la realización de la encuesta mediante cuestionario impreso, que se aplicó a los clientes quienes usufructuaban el servicio en los momentos de las visitas, que eran de dos a tres veces por día, también se le llamo a los clientes quienes están registrados en el hotel para pedir una encuesta, a quienes se le envió el formulario google drive, así se logró completar la muestra deseada. Una vez completado las 100 encuestas, se procesó los datos obtenidos apoyados en software SPSS para obtener los 
porcentajes de cada indicador, porcentaje general de cada dimensión y la calidad de servicio en general y se presenta en forma de cuadro a continuación.

\section{RESULTADOS Y DISCUSIÓN}

Tabla 1. Perfil de usuarios

\begin{tabular}{llcc}
\hline Indicadores & & $\mathbf{f . i}$ & $\mathbf{\%}$ \\
\hline \multirow{2}{*}{ Genero } & Masculino & 59 & $59 \%$ \\
& Femenino & 41 & $41 \%$ \\
\hline \multirow{3}{*}{ Grado de estudio } & Primario & 6 & $6 \%$ \\
& Secundario & 28 & $28 \%$ \\
& Universitario & 66 & $66 \%$ \\
\hline Frecuencia de & Primera vez & 32 & $32 \%$ \\
Visita & Segunda vez y más & 68 & $68 \%$ \\
\hline \multirow{2}{*}{ Motivo de visita } & Laboral & 61 & $61 \%$ \\
& Turismo & 20 & $20 \%$ \\
& Otros & 19 & $19 \%$ \\
\hline & \multicolumn{1}{c}{ Total } & $\mathbf{1 0 0}$ & $\mathbf{1 0 0 \%}$ \\
\hline
\end{tabular}

Fuente: encuesta a los usuarios del hotel Ysapy

La mayor cantidad de los encuestados pertenecen al género masculino, poseen estudios universitarios, ya han utilizado el servicio por más de una ocasión y la mayoría por motivos laborales.

Tabla $\mathbf{N}^{\circ} 2$ Calidad de Servicio. Aspectos tangibles

\begin{tabular}{|c|c|c|c|c|c|c|}
\hline Dimensión & Indicadores & Deficiente & Aceptable & Regular & Bueno & Excelente \\
\hline \multirow{5}{*}{$\begin{array}{l}\text { Elementos } \\
\text { tangibles }\end{array}$} & $\begin{array}{l}\text { Equipos tecnológicos } \\
\text { modernos }\end{array}$ & $11 \%$ & $29 \%$ & $6 \%$ & $28 \%$ & $26 \%$ \\
\hline & $\begin{array}{l}\text { Instalaciones fisicas } \\
\text { atractivas }\end{array}$ & $7 \%$ & $12 \%$ & $8 \%$ & $42 \%$ & $31 \%$ \\
\hline & $\begin{array}{l}\text { Uniforme adecuado y } \\
\text { apariencia pulcra de los } \\
\text { empleados }\end{array}$ & $17 \%$ & $19 \%$ & $6 \%$ & $44 \%$ & $14 \%$ \\
\hline & Publicidad atractivas & $17 \%$ & $33 \%$ & $11 \%$ & $25 \%$ & $14 \%$ \\
\hline & $\begin{array}{l}\text { Valoración total en \% } \\
\text { de elementos tangibles }\end{array}$ & $13 \%$ & $23 \%$ & $8 \%$ & $35 \%$ & $21 \%$ \\
\hline
\end{tabular}

Fuente: encuesta a los usuarios del hotel Ysapy

La tabla número 2 presenta la valoración de los aspectos tangibles, la mayor cantidad de encuestados coincidían que los elementos tangibles son buenos y entre los indicadores más deficientes encontramos, portar uniforme adecuado y la apariencia pulcra de los empleados y la falta de atracción de la publicidad sobre el hotel como folletos, banners y otros. 
Tabla N 3 Calidad de Servicio. Dimensión Fiabilidad

\begin{tabular}{|c|c|c|c|c|c|c|}
\hline Dimensión & Indicadores & Deficiente & Aceptable & Regular & Bueno & Excelente \\
\hline \multirow{6}{*}{ Fiabilidad } & $\begin{array}{l}\text { Capacidad para } \\
\text { atender pedido en } \\
\text { tiempo prometido }\end{array}$ & $3 \%$ & $8 \%$ & $17 \%$ & $44 \%$ & $28 \%$ \\
\hline & $\begin{array}{l}\text { Interés para resolver } \\
\text { problemas }\end{array}$ & $6 \%$ & $14 \%$ & $14 \%$ & $44 \%$ & $22 \%$ \\
\hline & $\begin{array}{l}\text { Capacidad de } \\
\text { mantener el local } \\
\text { desinfectado }\end{array}$ & $8 \%$ & $5 \%$ & $7 \%$ & $52 \%$ & $28 \%$ \\
\hline & $\begin{array}{l}\text { Concluir servicio en } \\
\text { tiempo prometido }\end{array}$ & $0 \%$ & $6 \%$ & $14 \%$ & $36 \%$ & $44 \%$ \\
\hline & $\begin{array}{l}\text { Brindar } \\
\text { informaciones } \\
\text { confiables }\end{array}$ & $3 \%$ & $8 \%$ & $14 \%$ & $28 \%$ & $47 \%$ \\
\hline & $\begin{array}{l}\text { Valoración total de } \\
\text { los indicadores de } \\
\text { la fiabilidad en \% }\end{array}$ & $4 \%$ & $8 \%$ & $13 \%$ & $41 \%$ & $34 \%$ \\
\hline
\end{tabular}

Fuente: encuesta a los usuarios del hotel Ysapy

La dimensión fiabilidad la mayoría coincidían que la dimensión de fiabilidad hace en bueno, pero hay puntos débiles que requieren ajustes para mejorar la calidad del servicio, como en específico el interés que muestran los empleados para resolver los problemas.

Tabla N$^{\circ} 4$ Calidad de Servicio. Dimensión Capacidad de respuesta

\begin{tabular}{|c|c|c|c|c|c|c|}
\hline Dimensión & Indicadores & Deficiente & Aceptable & Regular & Bueno & Excelente \\
\hline \multirow{5}{*}{$\begin{array}{l}\text { Capacidad } \\
\text { de } \\
\text { respuesta }\end{array}$} & $\begin{array}{l}\text { Comunicación } \\
\text { oportuna }\end{array}$ & $3 \%$ & $14 \%$ & $11 \%$ & $50 \%$ & $22 \%$ \\
\hline & Servicio rápido & $3 \%$ & $5 \%$ & $14 \%$ & $42 \%$ & $36 \%$ \\
\hline & $\begin{array}{l}\text { Disponibilidad de } \\
\text { respuesta }\end{array}$ & $0 \%$ & $11 \%$ & $2 \%$ & $31 \%$ & $56 \%$ \\
\hline & Resuelve reclamos & $0 \%$ & $11 \%$ & $20 \%$ & $47 \%$ & $22 \%$ \\
\hline & $\begin{array}{l}\text { Valoración total de } \\
\text { los indicadores de } \\
\text { la capacidad de } \\
\text { respuesta en \% }\end{array}$ & $1,50 \%$ & $10 \%$ & $12 \%$ & $42,5 \%$ & $34 \%$ \\
\hline
\end{tabular}

Fuente: encuesta a los usuarios del hotel Ysapy

La mayor cantidad de encuestados coincidieron que esta dimensión es buena, indica la necesidad de planes de mejora para elevar la calidad del servicio en los siguientes indicadores: la comunicación oportuna, disponibilidad de respuesta y resolución de reclamos

Tabla $\mathbf{N}^{\circ} 5$ Calidad de Servicio. Dimensión Seguridad 


\begin{tabular}{|c|c|c|c|c|c|c|}
\hline \multirow{5}{*}{ Seguridad } & $\begin{array}{l}\text { Comportamiento } \\
\text { confiables }\end{array}$ & $6 \%$ & $2 \%$ & $6 \%$ & $40 \%$ & $46 \%$ \\
\hline & $\begin{array}{l}\text { Seguridad en las } \\
\text { transacciones }\end{array}$ & $2 \%$ & $7 \%$ & $20 \%$ & $31 \%$ & $40 \%$ \\
\hline & Amabilidad & $2 \%$ & $7 \%$ & $11 \%$ & $20 \%$ & $60 \%$ \\
\hline & $\begin{array}{l}\text { Conocimiento } \\
\text { adecuados }\end{array}$ & $0 \%$ & $17 \%$ & $14 \%$ & $43 \%$ & $26 \%$ \\
\hline & $\begin{array}{l}\text { Valoración total de } \\
\text { los indicadores de } \\
\text { la seguridad en \% }\end{array}$ & $2,50 \%$ & $8 \%$ & $13 \%$ & $33,5 \%$ & $43 \%$ \\
\hline
\end{tabular}

\section{Fuente: encuesta a los usuarios del hotel Ysapy}

La mayor cantidad de encuestados coincidieron en calificar la dimensión seguridad como excelente. Se identifican indicadores que presenta observación como el conocimiento, entrenamiento y formación específica que deberían poseer los empleados

Tabla N $^{\circ} 6$ Calidad de Servicio. Dimensión Empatía.

\begin{tabular}{|c|c|c|c|c|c|c|}
\hline Dimensión & Indicadores & Deficiente & Aceptable & Regular & Bueno & Excelente \\
\hline \multirow{6}{*}{ Empatía } & $\begin{array}{l}\text { Atención } \\
\text { personalizada }\end{array}$ & $3 \%$ & $0 \%$ & $17 \%$ & $31 \%$ & $49 \%$ \\
\hline & Horario accesibles & $2 \%$ & $3 \%$ & $9 \%$ & $17 \%$ & $69 \%$ \\
\hline & $\begin{array}{l}\text { Empleados que } \\
\text { brinda atención } \\
\text { personalizada }\end{array}$ & $0 \%$ & $3 \%$ & $11 \%$ & $26 \%$ & $60 \%$ \\
\hline & $\begin{array}{l}\text { Preocupación por } \\
\text { los interese del } \\
\text { cliente }\end{array}$ & $0 \%$ & $8 \%$ & $17 \%$ & $49 \%$ & $26 \%$ \\
\hline & $\begin{array}{l}\text { Comprensión de } \\
\text { necesidades } \\
\text { especificas } \\
\end{array}$ & $0 \%$ & $4 \%$ & $19 \%$ & $44 \%$ & $33 \%$ \\
\hline & $\begin{array}{l}\text { Valoración total de } \\
\text { los indicadores de } \\
\text { la seguridad en \% }\end{array}$ & $1 \%$ & $4 \%$ & $15 \%$ & $33 \%$ & $47 \%$ \\
\hline
\end{tabular}

Fuente: encuesta a los usuarios del hotel Ysapy

La tabla numero 6 presenta los resultados de la dimensión de empatía, de los 100 clientes encuestados, respecto a los indicadores de la empatía respondieron que es excelente y el punto que requiere de una atención especial es la preocupación de los empleados por los intereses de los clientes.

\section{DISCUSIÓN}

La mayor cantidad usuarios encuestados presentan las siguientes características: pertenecen al género masculino, cuentan con formación nivel universitario, con más de un alojamiento en el servicio por motivos laborales y en menor proporción por motivos turísticos; datos que 
coinciden con caracterización de usuarios relatados por propietarios del servicio hotelero. Con respecto a la mayor cantidad de usuarios masculino el dato coincide con la tendencia estadística que indica que en la fuerza de trabajo económicamente activa en Paraguay los hombres son los más favorecidos(DGEEC,2020). La mayor cantidad de los encuestados han utilizado el servicio hotelero en más de una ocasión, esta situación estaría indicando la presencia de clientes habituales o en proceso de fidelización como resultado de aceptación, comodidad de usuarios que de acuerdo a Mesén Figueroa (2011) la fidelidad se produce cuando existe una situación ideal o favorable entre la expectativa del cliente y los servicios recibidos, por lo que facilita una relación duradera entre cliente y empresa.

La dimensión de aspectos tangibles en general obtuvo la calificación de bueno, identificando puntos más críticos que requieren mejorar como la falta de atracción de publicidad sobre el hotel como folletos, banners, etc y la apariencia pulcra y uniforme adecuado de los empleados, mientras que la dimensión de seguridad es excelente. Los aspectos tangibles y la seguridad son homogéneas, cumplen con las expectativas y satisfacción de los usuarios, la primera impresión de la empresa se genera cuando el cliente ingresa al establecimiento, y es importante garantizar una estancia agradable (Veloz Navarrete \& Vasco Vasco, 2016)

Por otra parte, en cuanto a la dimensión de fiabilidad; la mayor cantidad de encuestados respondieron que es bueno y el punto que requiere de una atención específica, ya que registran algunas críticas por parte de los clientes es el interés que demuestran los empleados para resolver los problemas. Es la capacidad que demuestra la empresa para cumplir con su promesa o la calidad prometida a los usuarios (Matsumoto Nishizawa, 2014), en las organizaciones eficientes para complacer a los clientes solo prometen lo que pueden ofrecer respecto a servicios de alojamiento, cuando el cliente decide usufructuar el servicio, el hotel ofrece un valor adicional que termina superando la expectativa de los clientes. (Castro Paccha \& Paccha Soto, 2018)

También en la dimensión de capacidad de respuesta es buena y los indicadores que son aceptables pero que requieren de mejoría para complacer al cliente son: la comunicación oportuna de parte de los empleados con los clientes, la disponibilidad de respuestas por parte de los empleados para los clientes y la capacidad de los empleados para resolver los problemas que se presentan. La capacidad de respuesta tiene que ver con acciones tomadas a base de los conocimientos obtenidos y habilidades desarrolladas que ayuda a una organización para comprender y actuar efectivamente (Liao et al.,2003, citado en Demuner 
Flores et al.,2020). Las empresas con rápida respuesta tienden a ser competidores superiores con agilidad, ingenio y capacidad de aprender de sus errores (Nonaka y Takeuchi,1995; Jansenet al., 2005 citado en Demuner Flores et al.,2020).

Por último, respecto a la dimensión de empatía de la calidad del servicio del hotel es excelente. La empatía significa que los empleados están en disposición de ofrecer cuidado y atención personalizada a sus clientes, más allá de la cortesía, requiere de compromiso e implicación con los clientes, conocimientos a fondo de sus características y necesidades personales y de sus requerimientos específicos (Cottle, 1991).

\section{CONCLUSIÓN}

Los usuarios del hotel Ysapy en mayor cantidad son de género masculino, con buen nivel educativo y con proceso de fidelización.

En promedio general los clientes del hotel Ysapy evaluaron la calidad del servicio con la calificación de bueno, por lo tanto, responde a las expectativas y necesidades adecuadas al perfil del cliente, en gran medida mantiene el carácter de fiabilidad en los servicios que ofrecen, hechos que se evidencia a su vez en la fidelización de la mayoría delos clientes consultados. "Los clientes tienen ciertas expectativas acerca de la calidad de los servicios antes de la compra basándose en sus necesidades, experiencias o indicciones" (Gadotti dos Anjos \& França de Abreu, 2008, p. 5).

También en estudio similar realizado por Molina Huaccache(2018) concluyó que en su mayoría los encuestados indicaron la calidad del servicio en dicho hotel, es bueno, lo cual se puede señalar que el hotel está brindando un servicio de calidad, generando percepciones positivas y obteniendo clientes satisfechos.

Por último, al concluir se recomienda al encargado del hotel Loreliz que tomen como práctica la aplicación de técnicas para evaluar la calidad del servicio de su hotel desde la perspectiva de los usuarios, que les permitirá ajustar las brechas existentes entre los servicios ofrecidos y las expectativas del cliente, y así lograr la satisfacción de los clientes.

\section{REFERENCIAS}

Daza Higuera, J. M. (2013). Análisis de la medición de calidad en los servicios hoteleros. Criterio Libre, .

Duque Oliva, E. J., \& Palacios Palacios, D. (2017). EVALUATION OF THE SERVICE QUALITY PERCEIVED IN THE. Criterio Libre • Vol. 15 - No. 26 • Bogotá (Colombia. 
Mesén Figueroa, V. (2011). Fidelizacion de clientes, concepto y perspectiva contable. . TEC Empresarial.

Rios, J., \& Santomá, R. (2008). Calidad de Servicio en la Industria Hotelera desde la perspectiva del. Management\& Empresa, 1-12.

Arias Muñoz, C. E. (2019). CALIDAD DEL SERVICIO Y SASTIFACCIÓN DEL CLIENTE EN LAS MYPEDEL SECTOR SERVICIOS RUBRO HOTELES DE 3 ESTRELLAS DEL DISTRITO DE PIURA ,2015. In Crescendo .

Carbajal Prieto, L. (s.f.). Análisis de la gestión de. Rev. esc.adm.neg. No. 76, 78-91.

Castro Paccha, E., \& Paccha Soto, M. (2018). INFLUENCE ON THE QUALITY OF SERVICE. Dialnet.

Cottle, D. W. (1991). El Servicio centrado en el Cliente. Obtenido de https://books.google.com.py/books/about/El servicio-centrado-en-elcliente.html?id=o37JeJiO-qoC

Demuner Flores, M. d., Becerril Torres , O. U., \& Ibarra Cisneros , M. A. (2020). Capacidad de respuesta y capacidad de absorsión. Nóesis. Revistas de ciencias sociales y humanidades .

DGEEC. (2020 de 09 de 2020). DGEEC publicó datos sobre la población joven del país. Obtenido de https://www.ine.gov.py/news/news-contenido.php?codnews=525\#: :text=41\%2C9\%25).,692.000\%20fueron\%20hombres\%20y\%20459.

Falces Delgado, C., Sierra Díez, B., Becerra Grande, A., \& Briñol Turnes, P. (1999). HOTELCUAL: UNA ESCALA PARA MEDIR CALIDAD PERCIBIDA EN SERVICIOS DE ALOJAMIENTO. Estudios Turísticos, n.139, 95-110.

Fernández Cristaldo, A. (2020). Clima organizacional y calidad de servicio ofrecido por la Universidad. Ciencia Latina revista Multidiciplinar , 3-18.

Gadotti dos Anjos, S. J., \& França de Abreu, A. (2008). La medición de la calidad de servicio: una aplicación en empresas hoteleras. Revista Europea de Dirección y Economía de la Empresa, vol. 18, núm. 2 (2009), pp. 175-18.

Girón España, N. E. (2016). Medición de la Calidad de servicio del Hotel Real Gran Chortí del municipio de Esquipulas a travez del modelo Servqual. 21-99.

GROWTH INSTITUTE. (2019). Dr. Leonard Berry. Obtenido de https://www.growthinstitute.com/es/faculty/dr-leonard-berry/ 
Instituto Nacional de Estadística. (2018). www.ine.gov.py. Obtenido de Neembucú Proyeccion de la población por Sexo y Edad, 2018.

Mansalve Castro , C., \& Hernández Rueda, S. (2015). GESTIÓN DE LA CALIDAD DEL SERVICIO EN LA HOTELERÍA. 160-173.

Matsumoto Nishizawa, R. (2014). Desarrollo del Modelo Servqual para la medición de la calidad de servicioen la empresas de publicidad Ayuda Experto. Revista Perspectiva. Matsumoto Nishizawa, R. (2014). Desarrollo del modelo servqual para la medicion de la calidad del servicipoen la empresa de publicidad Ayuda Experto. Perspectivas, 34.

Mesén Figueroa, V. (2011). Fidelización de Clientes: concepto yperspectiva contable. Tec Empresarial, 29-35.

Molina Huaccache, R. C. (2018). Nivel de calidad del servicio desde la percepción de los clientes del Hotel Llaqta, distrito de Miraflores, Lima, 2018”.

Morales, V., Hernández Medo, A., \& Blanco, Á. (2009). EVALUACIÓN DE LA CALIDAD EN ORGANIZACIONES DEPORTIVAS: ADAPTACIÓN DEL MODELO SERVQUAL. Revista de Psicologia del deporte 2009. Vol.18 .num,2.pp.137-150, 214.

Pacabaque, A. N., \& Rocha Buelvas, A. (2016). Modelos SERVQUAL y SERVQHOS para la evaluacion de calidad de los servicios de salud. Rev.Fac.Med.2016 Vol $64 \mathrm{~N}$ 4:715-20, 2-6.

Riveros Montiel, T., Olmedo de Ríos , S., Ventury, j., \& Gómez, J. M. (2019). TURISMO PARA EL DESARROLLO Potencialidades y desafíos en Ñeembucú. Obtenido de www.conacyt.gov.py

ROCKBRIDGE. (2021). Profesor A. Parasuraman. Obtenido de https://rockresearch.com/staff/professor-a-parasuraman/

Santamaría Escobar, Á. E., \& Cadrazco Parra, W. (2011). Matriz de Inteligencia Hotelera mih Una propuesta para el mejoramiento. Pensamiento \& Gestión, 211-246.

Senatur. (2018). enatur.gov.py/noticias/el-turismo-se-ubico-como-una-de-las-principalesactividades-economicas-en-los-ultimos-5-anos.

Senatur. (05 de 2020). senatur presenta a Misiones y Neembucú como destino de turismo interno post Covid 19. Obtenido de senatur.gov.py/noticias/senatur-presenta-aMisiones-y-Ñeembucú-como-destino-de-turismo-interno-post-Covid 19 
Senatur. (05 de 2020). senatur presenta a Misiones y Neembucucomo destinos de turismo interno post covid19. Obtenido de www.senatur.gov.py

Sharón, A., \& Weil, G. (2003). Medición de la Calidad de los Servicios. Obtenido de https://scholar.google.es/scholar?hl=es\&as_sdt=0\%2C5\&q=brechas+en+calidad+d e+servicios\&btnG=\#d=gs_qabs\&u=\%23p\%3DSMfid0gAD70J

Thompson, I. (2005). La Satifacción del Cliente. ACADEMIA, 2-7.

UNC. (2021). VALARIE ZEITHAML. Obtenido de Profesor adjunto de marketing: https://www.kenan-flagler.unc.edu/faculty/directory/valarie-zeithaml/

UNIVERSIDAD NACIONAL DE PILAR . (2021). unp.edu.py. Obtenido de www.unp.edu.py

Vavra, T. G. (2000). CÓMO MEDIR LA SASTIFACCIÓN DEL CLIENTE SEGÚN LA ISO 9001: $20002^{\circ}$ EDICIÓN. MADRID: @FUNDACIÓN CONFEMETAL.

Veloz Navarrete, C., \& Vasco Vasco, J. (2016). Quality in service of hotel companies of second category. Revista Ciencia UNEMI.

Wigodski Sirebrenik, J. (2003). What is SERVQUAL? Obtenido de https://www.medwave.cl/link.cgi/Medwave/Enfermeria/Nov2003/2763?ver=sindis eno 